NASA Technical Memorandum 107101

\title{
Integrated Cryogenic Satellite Communications Cross-Link Receiver Experiment
}

R.R. Romanofsky, K.B. Bhasin, and A.N. Downey

Lewis Research Center

Cleveland, Ohio

C.J. Jackson, and A.H. Silver

TRW

Redondo Beach, California

H.H.S. Javadi

Jet Propulsion Laboratory

Pasadena, California

Prepared for the

16th International Communications Satellite Systems Conference sponsored by the American Institute of Aeronautics and Astronautics Washington D.C., February 25-29, 1996

National Aeronautics and

Space Administration 


\title{
INTEGRATED CRYOGENIC SATELLITE COMMUNICATIONS CROSS-LINK RECEIVER EXPERIMENT
}

\author{
R.R. Romanofsky, K.B. Bhasin and A.N. Downey \\ National Aeronautics and Space Administration \\ Lewis Research Center \\ Cleveland, $\mathrm{OH} 44135$ \\ C.J. Jackson, A.H. Silver \\ TRW, \\ Redondo Beach, CA 90278 \\ H.H.S. Javadi \\ Jet Propulsion Laboratory \\ Pasadena, CA 91109
}

\begin{abstract}
An experiment has been devised which will validate, in space, a miniature, high-performance receiver. The receiver blends three complementary technologies: high temperature superconductivity (HTS), pseudomorphic high electron mobility transistor (PHEMT) monolithic microwave integrated circuits (MMIC), and a miniature pulse tube cryogenic cooler. Specifically, an HTS band pass filter, InP MMIC low noise amplifier, HTS-sapphire resonator stabilized local oscillator (LO), and a miniature pulse tube cooler will be integrated into a complete $20 \mathrm{GHz}$ receiver downconverter. This cooled downconverter will be interfaced with customized signal processing electronics and integrated onto the space shuttle's "HitchHiker" carrier. A pseudorandom data sequence will be transmitted to the receiver, which is in low Earth orbit (LEO), via the Advanced Communication Technology Satellite (ACTS) on a $20 \mathrm{GHz}$ carrier. The modulation format is QPSK and the data rate is $1.024 \mathrm{Mbps}$. The bit error rate (BER) will be measured in situ. The receiver is also equipped with a radiometer mode so that experiment success is not totally contingent upon the BER measurement. In this mode, the receiver uses the Earth and deep space as a hot and cold calibration source, respectively. The experiment closely simulates an actual cross-link scenario. Since the receiver performance depends on channel conditions, its true characteristics would be masked in a terrestrial measurement by atmospheric absorption and background radiation. Furthermore, the receiver's performance depends on its physical temperature, which is a sensitive function of platform environment, thermal design, and cryocooler performance. This empirical data is important for building confidence in the technology.
\end{abstract}

Introduction

The benefit of cryogenic cooling to low noise receiver front-ends has been well established. For example, the noise temperature of a Ka-band PHEMT amplifier can be reduced an order of magnitude by lowering the physical temperature of the device from $300 \mathrm{~K}$ to $20 \mathrm{~K} .1,2$ The satellite communications community has generally recoiled from serious consideration of this technology because onboard cooling appeared unrealistic. For example, to provide one Watt of cooling at $77 \mathrm{~K}$, about $1600 \mathrm{~kg}$ of liquid nitrogen would be necessary to support a ten year mission life. At the communications subsystem level, passive cooling cannot supply the required lift at the desired operating temperature. However, recent advances in mechanical refrigerators cause us to reevaluate this issue. Single-stage, closed-cycle coolers are available that: provide ample cooling capacity in the neighborhood of $77 \mathrm{~K}$; offer a mean time between failures (MTBF) of order 10 years; and consume only about $30 \mathrm{~W}$ of prime power. Coupled with the late discovery of high temperature superconductors (HTS), and the emergence of PHEMT MMIC, cooled front ends become practical and enticing.

The surge in wireless communications including voice, video, and data creates a tremendous demand for channel bandwidth and drives transceiver systems to frequencies beyond $20 \mathrm{GHz}$. In some cases, terrestrial wireless networks are challenging the satellite industry for precious frequency spectrum allocation. Bandwidth will necessarily be rationed. Terrestrial and space-based systems will exact the final measure of performance from state-of-the-art technologies to be competitive and provide the desired quantity and quality of services. So, it is not too speculative to presume that these pressures will encourage the 
consideration of technical approaches that might once have been regarded as too high-risk.

Link performance depends on frequency, antenna aperture, transmitter power, range, and receiver sensitivity or noise figure. Technological maturity, space-flight dynamics, and spacecraft resources place constraints on these parameters. Reducing the receiver noise figure may be the most cost effective way to improve, and in some cases enable, advanced microwave and millimeter-wave commercial satellite links. The same is true for at least one NASA application. Consider a $32 \mathrm{GHz}$ Mars-to-Earth relay satellite link at $10 \mathrm{Mbps}$. Assume a present technology $110 \mathrm{~W}$ traveling wave tube (TWT) transmitter. To close such a link would require a receiver noise figure $(0.5 \mathrm{~dB})$ achievable only through cryogenic cooling, even with 10 and 20 meter transmitter and receiver antennas, respectively. 3 High data rate cross-links for the next generation of satellite systems would benefit likewise. A cross-link employing a cooled receiver, not unlike the one described here, would use less overall spacecraft power since transmitter power could be conserved. As an example to illustrate the benefit crossover, consider a hypothetical Ka-band cross-link requiring $10 \mathrm{~W}$ of transmitter power and assume the transmitter is 15 percent efficient. (This is a state-of-the-art TWT, but $>30$ percent efficient TWTs are in development. ${ }^{4}$ ) If the noise power at the receiver could be reduced by a factor of 2 without costing more than about $30 \mathrm{~W}$ of prime power, there would be a system advantage. High data rate satellite links, historically a haven for TWTs, could be established with small, lightweight solid state power amplifiers (SSPA). This paper discusses a practical receiver architecture emphasizing sensitivity and miniaturization, and will further outline an experiment for validating the required technology.

\section{Experiment Methodology}

The experiment will demonstrate that a K-band superconducting/semiconducting receiver, operating at a temperature in the neighborhood of $75 \mathrm{~K}$, can economically and dramatically: increase data rates, or decrease antenna aperture, or reduce transmitter power. The K-band low noise receiver (LNR) is located on a LEO platform (i.e., the space shuttle) which receives transmissions from ACTS. The use of ACTS provides a timely, low cost experimental cross-link. The up and down ACTS links are $30 \mathrm{GHz}$ and $20 \mathrm{GHz}$, respectively. Parameters to be measured (BER and noise figure) are functions of the communications channel conditions, including atmospheric absorption and background radiation. The benefits of an ultra low noise receiver are masked if data is transmitted via a link through the atmosphere, where it is well known that noise temperatures may approach
$290 \mathrm{~K}$. (It is important to realize that a cross-link demonstration at $20 \mathrm{GHz}$ supports future $60 \mathrm{GHz}$ crosslinks and $30 \mathrm{GHz}$ deep-space relays, and the cryopackaging approach is essentially independent of frequency.) The selected demonstration frequency of 20 $\mathrm{GHz}$ is especially troublesome since a water vapor emission line exists here. Diffracted thermal emission from the ground (i.e., a $290 \mathrm{~K}$ source) interferes with system performance as well.

Total system noise involves two elements: the intrinsic receiver noise figure and noise captured by the antenna. The antenna main beam looking through the atmosphere coupled with antenna back and side lobes contributes unspecified noise which renders a precise determination of intrinsic receiver noise impossible. Since the background noise temperature of deep-space is approximately $2.7 \mathrm{~K}$, system noise for the experiment (and ultimately the application) will be dominated by the inherent receiver noise. 5 Furthermore, since performance is a sensitive function of the integrated package physical temperature, which is influenced by the platform environment, the only way to quantify LNR performance accurately is to conduct the experiment in space.

Two independent measurements will be performed to evaluate the performance of the LNR. The most general indicator of a digital communications system's performance is the BER. Normally, bit error detection is achieved by comparing a delayed version of the data with the demodulated data. In this experiment, the original data is not available at the demodulator. A polynomial error detector will be used. A pseudo random message will be generated and sent from NASA Lewis in Cleveland, Ohio to the ACTS satellite in geostationary orbit at $100^{\circ} \mathrm{W}$ longitude. ACTS translates the signal and retransmits it at $20 \mathrm{GHz}$ to the orbiter. The uplink signal-to-noise ratio can be made arbitrarily large by increasing transmitter power; hence, the uplink will not degrade the experiment. This portion of the experiment is referred to as the communications mode. That is, the receiver antenna is pointed to ACTS and a BER test is performed.

In principle, the system noise temperature could be determined by measuring the output power when a (hypothetical) matched source at $0 \mathrm{~K}$ is connected to the input. Likewise, if two sources at sufficiently different temperatures are available, the "Y"-factor technique can be used. 6 By pointing the receiver antenna toward Earth, the noise power $\mathrm{kT}_{\mathrm{h}} \mathrm{B}_{\mathrm{r}}$ can be measured. Here, $\mathrm{k}$ is Boltzmann's constant, $\mathrm{B}_{\mathrm{r}}$ is the predetection bandwidth, and $\mathrm{T}_{\mathrm{h}}$ is the equivalent noise temperature of a "hot" Earth. Then the antenna is pointed toward empty space to measure the noise power of a "cold" source, $\mathrm{kT}_{\mathrm{c}} \mathrm{B}_{\mathrm{r}}$, where $\mathrm{T}_{\mathrm{c}}$ is the background temperature of "cold" space. The power received by a square law detector for the hot and cold cases are designated $\mathrm{P}_{h}$ and $\mathrm{P}_{c}$, respectively. Let $\mathrm{Y}=\mathrm{P}_{\mathrm{h}} / \mathrm{P}_{\mathrm{c}}$, then 
it is easily shown that $T_{e}=\left(T_{h}-Y T_{c}\right) /(Y-1)$ where $T_{e}$ is the noise temperature of the receiver. This is referred to as the radiometer mode. The relationship expressing BER as a function of $\mathrm{Eb} / \mathrm{No}$, which is the ratio of the energy-perbit to noise power-per-bandwidth, is well known. Furthermore, $\mathrm{Eb} / \mathrm{No}=\mathrm{S} / \mathrm{N}(\mathrm{B} / \mathrm{R})$ where $\mathrm{S} / \mathrm{N}$ is the signalto-noise ratio, $\mathrm{B}$ is the information bandwidth, and $\mathrm{R}$ is the data rate. Hence, the noise temperature measurement serves to corroborate the BER measurement. (The oscillator phase noise can be measured during ground tests, and as long as the receiver physical temperature is known, the phase noise can be accounted for along with the noise figure to predict the BER.)

The preferred platform for the experiment is the space shuttle for the following reasons: it mimics the conditions experienced by a typical LEO satellite, it provides readily available resources (power, thermal, telemetry), and it provides a critical pointing function otherwise obtainable only at substantially added cost and complexity. The experiment will be executed when the orbiter is located between $115^{\circ} \mathrm{W}$. and $85^{\circ} \mathrm{W}$., with a typical $28.5^{\circ}$ inclination. Under these conditions, the magnitude of the Doppler shift will not exceed $200 \mathrm{kHz}$ and the rate will be less than $600 \mathrm{~Hz} / \mathrm{s}$. During this interval, the orbiter will traverse the beam of the 1 meter steerable antenna on ACTS. The BER measurement sequence will be completed in less than $80 \mathrm{~s}$. Functional requirements impose upper and lower limits on link duration in the following way. For statistical relevance, it is desirable to acquire at least 100 errors to determine the BER. So a lower limit on BER is about 5 X 10-6 because of experiment duration. An upper permissible limit on BER is about $10^{-3}$ because of potential demodulator synchronization problems. It is desirable to perform the experiment when the orbiter is eclipsed. (i.e., when it is night time in the western hemisphere.) Otherwise, the sun could corrupt the measurement by adding unwanted noise through either the main beam or sidelobes. Even though the sun subtends a small solid angle compared to the entire antenna main beamwidth, $\mathrm{T}_{\text {sun }}$ is calculated to be $10.3 \times 10^{3} \mathrm{~K}$ at $20 \mathrm{GHz}$ (assuming no flares), and the antenna temperature would greatly exceed the preferred temperature.7

During the $80 \mathrm{sec}$ [communications mode] link, the orbiter must be pointing the LNR antenna at ACTS. The orbiter is capable of maintaining a specified celestial or Earth reference attitude for payload pointing. The flight control system provides a half-cone angle stability of $+/-0.1^{\circ}$ per axis and a stability rate of $+/-0.01^{\circ}$ per sec per axis, using vernier thrusters. Assuming rotation about the vector is unimportant, the total pointing error should not exceed $1.3^{\circ}$ plus a mounting alignment uncertainty, due to mechanical and thermal tolerances, of $2^{\circ}$ maximum. The steerable antenna on ACTS is linearly polarized but a circularly polarized conical horn will be used for the receiver. This eliminates the need to have the orbiter perform an additional z-axis (yaw) maneuver. (The short link duration minimizes orbiter acrobatics. An entire BER test sequence can be completed following an initial 2-axis, pitch and roll, maneuver. That is, the satellite should remain in view for the necessary duration without continuous attitude adjustment.) Hence, there is an automatic but manageable $3 \mathrm{~dB}$ compromise in the link budget from polarization loss. The choice of a $10^{\circ}$ antenna beamwidth is based on a compromise between these considerations and the overall system gain/noise temperature ratio.

Ideally, for the radiometer mode, the antenna is briefly pointed to a celestial pole for the "cold" reference noise measurement. It is well known that the spectrum of the cosmic background radiation is that of a blackbody; therefore, it serves as an excellent calibration source. The radiometer measurement takes about $1 \mathrm{sec}$ and can occur anytime during the $1.5 \mathrm{hr}$ period between BER measurements. It will be assumed that the source is the north galactic pole, known to have a temperature of $2.735+/-0.06$ K.5 A relatively hot source, subtending a sufficiently large angle, could violate this premise. Hence, it is necessary, as a minimum, to point the antenna away from the ecliptic. The "hot" reference noise measurement will be made with the antenna pointed toward Earth, the usual shuttle orientation. (The orbiter will be required to travel in a nose forward, payload bay to space vector for approximately $2 \mathrm{hr}$. The orbiter can rotate at a rate of $0.2^{\circ}$ per sec so one can expect that the minimum time required to execute a $180^{\circ}$ roll is $15 \mathrm{~min}$. If $2 \mathrm{BER}$ measurements are to be made, the total elapsed time is about $2 \mathrm{hr}$, well within the flight rule restriction of $48 \mathrm{hr}$ cumulative.) The cartoon of Fig. 1 illustrates the experiment scenario.

An optional calibration measurement requires the receiver to be placed in a hybrid mode. In this mode, the antenna is pointed towards ACTS but the IF switch is connected to the radiometer electronics. The well characterized signal from ACTS can be used as an additional calibration source to determine system gain. This measurement requires that $S_{i}>>k T_{b} B$, where $S_{i}$ is the signal power from the satellite and $\mathrm{T}_{\mathrm{b}}$ is the apparent background temperature. So, the sun and moon should be out of the picture. Monitoring system gain variation, by recording detected power fluctuations, permits a better radiometric noise figure measurement.

Ground based command and control of the experiment will be accomplished via a 1200 baud asynchronous data link provided by the "HitchHiker" carrier avionics system. Just prior to each of the (nominally) two orbital passes a command will be issued to the experiment via the asynchronous uplink. The command will instruct the payload to clear the BER test set, and to set the value of an IF attenuator, which is initially set to zero. The BER will 
be measured every $10 \mathrm{sec}$ or so during the $80 \mathrm{sec}$ long pass through the beam of the 1 meter steerable antenna from ACTS. Mission elapsed time and orbiter state vector/ attitude data will help time- and space-stamp the BER data. There is a nominal transport delay (2 to $20 \mathrm{sec}$ for the uplink and 5 to $15 \mathrm{sec}$ for the downlink) between the GSFC customer ground support equipment and the payload, due to latencies introduced by the number of users issuing commands, Johnson Space Center mission control uplink delays, etc.

\section{Design Approach}

\section{$\underline{\text { X-Band Prototype }}$}

Several synergistic technologies have converged, in the sense of technological maturity, almost simultaneously. Noise temperatures of HEMTs cooled below liquid nitrogen are approaching the performance of maser amplifiers. (Higher carrier mobility from reduced phonon and impurity scattering in the undoped channel results in a much larger transconductance. The minimum noise figure scales as the reciprocal of the transconductance. Gate resistance is also smaller.) Compact, low loss HTS filters and HTS resonator stabilized oscillators, impossible before 1987, have been demonstrated. 8 The LNR under development here builds on our experience with a prototype X-band LNR demonstrated by NASA Lewis Research Center and the Jet Propulsion Laboratory. 9 This prototype LNR was developed in response to the Naval Research Laboratory's HTSSE-II program. 10 The HTSSE-II unit consists of an HTS pre-select filter, a two-stage hybrid HEMT low noise amplifier (LNA), a silicon diode mixer, and an FET feedback oscillator (LO) using an HTS resonator. The complete downconverter measures about $5.5 \times 11.0 \times 1.6$ $\mathrm{cm}^{3}$. The 4-pole microstrip filter has a pass-band loss of about $0.25 \mathrm{~dB}$ and measures $15.0 \times 7.0 \times 0.5 \mathrm{~mm}^{3}$. The center frequency is $7.35 \mathrm{GHz}$ and the $3 \mathrm{~dB}$ bandwidth is $400 \mathrm{MHz}$. The first stage of the LNA is optimized for noise figure at cryogenic temperatures and the second stage provides flat gain response. The LO is a GaAs MESFET based reflection mode hybrid circuit stabilized by an HTS linear resonator. 11 The output power and frequency are about $0 \mathrm{dBm}$ and $8.4 \mathrm{GHz}$, respectively. The balanced mixer is designed to operate with a "starved" LO.12 Since all the circuits are integrated into one package and cooled, it is necessary to minimize LO drive to reduce thermal demands on the cooler. The entire unit consumes only about $70 \mathrm{~mW}$, and provides a noise figure of $0.7 \mathrm{~dB}$ with $18 \mathrm{~dB}$ of gain at an operating temperature of $77 \mathrm{~K}$. Each submodule is attached to flat Ni/Au plated kovar carriers using silver filled epoxy. All submodules are screwed into a Ni/Au plated kovar housing which is hermetically sealed. A 0.001" layer of indium foil between each submodule and the floor of the housing provides good thermal contact. A photograph of the flight qualified downconverter is shown in Fig. 2.

\section{$\underline{20 \mathrm{GHz} \text { Integrated LNR }}$}

The downconverter design of the $20 \mathrm{GHz}$ receiver is basically an extension of the HTSSE-II X-band unit. Rather than applying each core technology at every opportunity, the downconverter selectively uses each where the benefit is substantial. For instance, in the integrated microwave assembly, HTS lines are used for the filter, but not for the mixer where line loss is not significant. The HTS filter is simulated based on staggered half-wavelength resonators using IE-3D software from Zeland Software, Corp. The center frequency is $19.53 \mathrm{GHz}$ with a $3 \mathrm{~dB}$ bandwidth of $200 \mathrm{MHz}$. A pass-band loss of $0.2 \mathrm{~dB}$ with a $20 \mathrm{~dB}$ return loss is obtained as a result of simulation. The filter will be fabricated from YBaCuO HTS films on a $\mathrm{LaAlO}_{3}$ substrate. The amplifier uses InP pseudomorphic ( $\mathrm{In}_{0.6} \mathrm{Ga}_{0.4} \mathrm{As}$ channel) HEMTs. The wafers will be grown using $\mathrm{MBE}$ and 0.1 micron gates will be fabricated using $\mathrm{Pt} / \mathrm{Ti} / \mathrm{Pt} / \mathrm{Au}$ metallization. A $0.6 \mathrm{~dB}$ noise figure is anticipated. The InP MMIC uses a factor of 3 less power than an analogous GaAs MMIC. Noise temperature data for a $20 \mathrm{GHz}$ GaAs MMIC, which will serve as a backup device for this experiment, is shown in Fig. 3.

The LO uses a $20.7 \mathrm{GHz} \mathrm{TE}_{011}$ mode HTS-sapphire resonator which has a loaded $\mathrm{Q}$ of about 105 . A two-stage GaAs amplifier with $>10 \mathrm{~dB}$ gain and $<4 \mathrm{~dB}$ noise figure has been selected. Phase shift in the feedback loop is accomplished by bonding 10 mil gold ribbons across a series of pads. The output matching circuit and phase shifter circuit are soldered to a gold plated $\mathrm{Cu} / \mathrm{W}$ carrier which has a thermal expansion coefficient matched to the substrates. A summary of downconverter specifications are shown in Table 1.

Possible system benefits from high-Q resonator stabilized oscillators should not be underestimated. The effect of oscillator phase noise on phase modulated digital communications systems, especially sensitive QPSK and higher formats, is fairly well understood.13,14 A model for the noise spectrum of a feedback oscillator has also been presented by Leeson.15 If a few simplifying assumptions are made, it is possible to gauge the effect of resonator $Q$ on BER performance directly, using closed form expressions. The derivation is straightforward but lengthy, and will not be presented here. Adapting Leeson's model, it is assumed that the power spectral density decreases with offset frequency as: $0 \mathrm{~dB} /$ octave past the feedback loop half-bandwidth $\left(\omega_{\mathrm{o}} / 2 \mathrm{Q}\right)$ and equal to the white noise floor; $6 \mathrm{~dB}$ per octave from the point where $1 / \mathrm{f}$ effects are no longer dominant; and $9 \mathrm{~dB} /$ octave from the carrier loop tracking bandwidth to the same point. From these (perhaps 
brazen) assumptions, a phase noise profile can be constructed in relation to Q. The RMS single sideband phase noise variance can then be obtained by integrating the spectral density profile over a specific offset frequency range. This range extends from the receiver carrier tracking loop cutoff frequency on the low end, to the receiver bandpass filter bandwidth on the high end. Then, a Gaussian probability density function for phase error is assumed. Typical results are shown in Fig. 4. The degraded BER curve corresponds to a $20 \mathrm{GHz}$ oscillator which had a loaded resonator $\mathrm{Q}$ of 250. This is about the best $\mathrm{Q}$ one could hope for from a metallic microstrip resonator. Phase noise was integrated from 102 to $10^{7} \mathrm{~Hz}$ and output power was $1 \mathrm{~mW}$. For a $\mathrm{Q}$ in excess of about $10^{4}$, no degradation was observed. While dielectric resonator oscillators can provide Q's of this order, certain HTS designs, like the one used in this experiment, may do even better. As always, the cost versus benefit ratio is application specific.

The signal processor block, Fig. 5, consists of a QPSK demodulator and Viterbi decoder printed circuit board (STEL-9236) and a BER counter. The STEL-9236 is designed and fabricated by Stanford Telecom. The demodulator can continuously tune from 950 to 1450 MHz. Coding gain is about $5 \mathrm{~dB}$ at $10^{-5} \mathrm{BER}$. In the communications mode, an industry standard polynomial code (CCITT) is sent to ACTS and relayed to the LNR onboard the orbiter. The STEL-9236 will demodulate and decode the received bits. The data checker detects errors and increments the error counter. Finally, the number of bits and the number of errors are stored into non-volatile active RAM and non-volatile passive RAM. This data is also transmitted to the Goddard Space Flight Center (GSFC), using an asynchronous downlink for a subsequent simple computation of BER. The pattern synchronizer, data checker, counters, and polynomial code generator are designed using field programmable gate array technology to minimize power, size, and weight.

In the radiometer mode, noise temperatures are read and the results stored in the on-board RAM and relayed to GSFC. A simple total power radiometer is used to measure receiver noise figure. The dynamic range of the detector diode is limited on the low end by sensitivity (TSS) and at the high end by non square law behavior and eventually burnout. Off-the-shelf Schottky diodes can provide a minimum detectable power of $-54 \mathrm{dBm}$. (Tunnel diode detectors are available which provide an extended TSS to $-60 \mathrm{dBm}$.) At a background temperature of $2.7 \mathrm{~K}$, the received power in a $200 \mathrm{MHz}$ bandwidth $\left(\mathrm{B}_{\mathrm{r}}\right)$ is about -117 $\mathrm{dBm}$. At a source temperature of $290 \mathrm{~K}$, the received power is $-96 \mathrm{dBm}$. The received signal is amplified and routed to the detector. Sensitivity is proportional to $\mathrm{T}_{\text {sys }} /$ $B_{r} 1 / 2$, where $T_{\text {sys }}$ is the receiver noise temperature. The final amplifier employs a matched diode to compensate for possible physical temperature variations in the detector.
A corrugated horn with a polarizer will be coupled to the LNA via a waveguide-to-coax transition. Flight hardware will be packaged in a "get away special" (GAS) canister with a motorized door to expose the receiver to space (Fig. 6). The antenna is designed so that the door poses no interference. (The door swings open $110^{\circ}$ from horizontal.) The GAS can, which provides a working volume of $5 \mathrm{ft}^{3}$, also serves as the containment vessel. It is interfaced with a cross-bay mounted "HitchHiker" carrier.

Of the three core and complementary technologies driving this experiment, cryocooler technology is the least understood by the satellite communications community. Cryogenic coolers for use on satellites require low power, long lifetime, and low weight. The need for exceptional reliability in a space cooler is even more critical on small satellites since cooler redundancy is often not an option due to weight constraints. Cryocooler costs are being driven down to meet IR sensor and high speed computing applications. The vibrationally balanced miniature pulse tube cooler, shown in Fig. 7, weighs $2.2 \mathrm{~kg}$ and delivers a maximum cooling power of $400 \mathrm{~mW}$ at $70 \mathrm{~K}$ for an input power to the compressor and drive electronics of $30 \mathrm{~W} .16$ The cooler can be balanced to reduce vibration forces below $0.1 \mathrm{~N}$ from 0 to $1000 \mathrm{~Hz}$ in all three axis. The cooler has passed launch vibration tests, and the MTBF is expected to be of order 10 years based on ongoing life tests. For both the Stirling and pulse tube cryocoolers, an electromagnetically driven piston operates as an oscillating pressure wave generator. Heat is pumped by the oscillating helium gas working fluid. A pretuned orifice connected to the gas reservoir passively controls the magnitude and phase of the mass flow relative to the phase of the almost sinusoidal pressure variation. The pulse tube, an empty piece of pipe, performs the required thermodynamic function of providing a mechanism through which PV work can be exhausted and allows a thermal gradient to be supported between the cold head and the orifice heat exchanger. A small clearance between the (non-contacting) piston, which compresses the gas, and the cylinder seals the compression space. The compressor operates at a frequency of $55 \mathrm{~Hz}$. The vibration balancer contained in the compressor pressure vessel operates $180^{\circ}$ out of phase with the compressor to cancel the force of imbalance created by the compressor motion. A pulse tube cooler can be cooled down rapidly compared to a Stirling cooler since at ambient temperature input power to the cooler is not limited by displacer dynamics. The pulse tube cooler has been flight qualified by subjecting it to a $14 \mathrm{~g}$ launch vibration spectrum.

Feed-throughs to the cryo-package are a new coaxial cable developed by Superconductor Technologies, Inc. These cables provide attenuation of only about $0.075 \mathrm{~dB} / \mathrm{cm}$ at $10 \mathrm{GHz}$ while offering a thermal conductance about 200 times smaller than an equivalent 
all copper cable. The microwave loss of low thermal conductivity stainless steel cables is quite high and makes them unattractive for an LNA feed. (The thermal conduction heat load to the cold stage is proportional to the product of conductor cross section and the material thermal conductivity. Ohmic heating is inversely proportional to cross sectional area. Vacuum separation and shielding are generally used to reduce radiation heating. ) The overall thermal budget for the LNR is shown in Table II. A thermal margin of 23 percent is provided. Although payload bay temperatures can range over the extremes of -70 o $\mathrm{C}$ to +40 o $\mathrm{C}$, a steady state temperature can be obtained with adequate thermal isolation. The entire receiver dissipates about $40 \mathrm{~W}$. Cooldown time is expected to be close to $24 \mathrm{hr}$.

\section{Impact on Satellite Systems and Ground Terminals}

Cryo-receivers offer more than just a successive refinement to the way satellites operate. Should the push for data rates approaching gigabits per second continue, they can provide an entirely new and economically tenable solution because of little added weight and volume. Of course, the radio astronomy community has been comfortable with cryogenically cooled sensors for three decades. There is also a conspicuous momentum building in other areas of cryogenic electronics for potential applications such as super-computers. It is anticipated that $77 \mathrm{~K}, 1 \mathrm{~W}$ coolers for ground applications will cost about $\$ 1500$ within a few years. Coolers are already available that can provide $0.25 \mathrm{~W}$ of cooling at the much more interesting temperature of $5 \mathrm{~K}$ for less than $\$ 18,000$. But it is really the synergy among HTS, InP MMICs, and cryocoolers that compels us to investigate space communications applications. Adapting the satellite to accomodate the technology doesn't seem particularly difficult. Thermal design is an essential part of any spacecraft and cryocoolers have been successfully integrated onto scientific satellites in the past. Cooler mass could be partially offset by incorporating an HTS microstrip filter, as was done here, instead of a waveguide version. HTS multiplexers are being developed which promise a considerable net mass savings. 17 Although circuit $\mathrm{Q}$ scales as 1/f for a superconductor, as opposed to $\mathrm{f}^{1 / 2}$ for a normal metal, superconductors still have an edge at nearly all the forseeable frequencies of interest. For intersatellite link applications, cooler power is largely offset by reduced transmitter power requirements. Thermal design of the spacecraft may need to be reconsidered in order to establish an equilibrium temperature closer to a preferred (i.e., <100 K) rather than a convenient (e.g., $290 \mathrm{~K})$ value. Solar shielding and management of waste heat will require more attention.
The composite technology may also benefit ground terminals, especially as the wireless revolution saturates the microwave frequency spectrum. As a perhaps somewhat optimistic example, consider the proposed Teledesic network. Their terminal-to-satellite link (TSL) calculations assumed a receiver noise figure of $2.5 \mathrm{~dB}$ at $20 \mathrm{GHz} .18$ If it is conservatively assumed that the noise figure can improve to $1.3 \mathrm{~dB}$, and if $1 / 3$ of the satellite TSL antennas are on-line, the savings exceeds $500 \mathrm{~W}$ per satellite.

The LNR experiment, sponsored by NASA's InSpace Technology Experiments Program office, is expected to be launched early in 1998 .

\section{Acknowledgements}

We would like to thank the Ohio Aerospace Institute, and in particular Mike Knasel and George Ziga, for administrative assistance and guidance during the evolution of this space experiment. We are also grateful to Denis Connolly, who instigated and helped define the experiment. Also, we recognize important contributions to component designs from Nam Nguyen, Nitin Soni, and Felix Miranda (NASA Lewis), Dennis Lo and Jeffrey Raab (TRW), David Miller (NYMA), and Zhi-Yuan Shen (DuPont).

\section{$\underline{\text { References }}$}

1. Pospieszalski, M. W., Nguyen, L. D., Lui, M., Liu, T., Thompson, M. A., Delaney, M. J., "Very low Noise and Low Power Operation of Cryogenic AlInAs/ GaInAs/InP HFET'S," IEEE MTT-S Digest, 1994, pp. 1345-1346.

2. Lai, R., Bautista, J. J., Fujiwara, B., Tan, K. L., Ng, G. I., Dia, R. M., Streit, D., Liu, P. H., Freudenthal, A., Laskar, J., Pospieszalski, M. W., "An Ultra-Low Noise Cryogenic Ka-Band InGaAs/InAlAs/InP HEMT Front-End Receiver," IEEE Microwave and Guided Wave Letters, Vol. 4, No. 10, October, 1994, pp. 329-33.

3. Ponchak, D. S., Zuzek, J. E., Whyte, W. A., Spence, R. L., Sohn, P. Y., "A Technology Assessmentof Alternative Communications Systems for the Space Exploration Initiative," NASA Lewis Research Center, Cleveland, Ohio, July 20, 1990.

4. Curren, A., Dayton, J., Palmer, R., Force, D., Tamashiro, R., Wilson, J., Dombro, L., Harvey, W., "A Low-Power, High Efficiency Ka-Band TWTA," 14th AIAA International Communication Satellite Systems Conference, Washington, DC., March, 1992. (AIAA-92-1822)

5. Mather, J.C., Cheng, E.S., Eplee, R.E., Isaacman, R.B., Meyer, S.S., Shafer, R.A., Weiss, R., Wright, E.L., Bennet, C.L., Boggess, N.W., Dwek, E., Gulkis, S., Hauser, M.G., Janssen, 
M., Kelsall, T., Lubin, P.M., Moseley, S.H., Murdock, T.L., Silverberg, R.F., Smoot, G.F., and Wilkinson, D.T., "A Preliminary easurement of the Cosmic Microwave Background Spectrum by the Cosmic Background Explorer (COBE) Satellite," The Astrophysical J., Vol. 354,May, 1990, pp. 37-40.

6. D.M. Pozar, Microwave Engineering, AddisonWesley Publishing Company, Inc., 1990, pp. 588-589.

7. Bokulic, R. S., "Use Basic Concepts to Determine Antenna Noise Temperature," Microwaves \& RF, March, 1991, pp. 107-115.

8. Shen, Z-Y, High-Temperature Superconducting Microwave Circuits, Artech House, Inc., Norwood, MA, 1994.

9. Javadi, H. H. S., Bowen, J. G., Chew, W., Rascoe, D. L., Romanofsky, R. R., Chorey, C. M., Bhasin, K. B., "JPL/NASA LeRC Space Qualified Hybrid High Temperature Superconducting/ Semiconducting 7.4 GHz Low-Noise Downconverter for NRL HTSSE-II Program," IEEE Trans. MTT, Vol. 43, No. 12, Dec. 95.

10. Nisenoff, M., Ritter, J. C., Price, G., Wolf, S. A., "The High Temperature Superconducting Space Experiment: HTSSE Icomponents and HTSSE IISubsystems and Advanced Devices," IEEE Trans. Applied Superconductivity, Vol. 3, No. 1, March, 1993, pp. 2885-2890.
11. Miranda, F. A., Chorey, C. M., Romanofsky, R. R., Bhasin, K. B., "Space Qualified Hybrid Superconductor/Semiconductor Planar Oscillator Circuit," Low Temperature Electronics and High Temperature Superconductivity Symposium, Reno, NV., May, 1995 (NASA TM-106978).

12. Romanofsky, R. R., "An X-Band Mixer Engineered for 77 K Operation," NASA TP-3538, A u gu s t, 1995.

13. Cheah, Y. C., "Analysis of Phase Noise in Oscillators," RF Design, November, 1991, pp. 99-104.

14. Howald, R. L., "Gauge Oscillator Spectral Density and Noise Functions," Microwaves \& RF, March, 1994, pp. 113-120.

15. Leeson, D. B., "A Simple Model of Feedback Oscillator Noise Spectrum," IEEE Proceedings Letters, February, 1966, pp. 329-330.

16. Chan, C. K., Jaco, C. B., Raab, J., Tward, E., Waterman, M., "Miniature Pulse Tube Cooler," 7th International Cryocooler Conference, 1992.

17. Mansour, R. R., Tang, W. C., Kudsia, C., "Superconductive Multiplexers for Statellite Payloads," AIAA-94-0987-CP, 1993.

18. "Characteristics of Teledesic's Ka Band, Low Earth Orbit, FSS Network to Provide Global Voice, Data, and Video Communications," Information Paper, June, 1992, Teledesic Corporation, Kirkland, WA. 
TABLE 1.-DOWNCOVERTER SPECIFICATIONS

\begin{tabular}{|c|c|c|c|}
\hline & Parameter & Specification & Goal \\
\hline LNA & $\begin{array}{l}\text { Gain }(\mathrm{dB}) \\
\text { Noise figure }(\mathrm{dB}) \\
\text { Center frequency } \\
3 \mathrm{~dB} \text { bandwidth } \\
1 \mathrm{~dB} \text { comp point } \\
\text { Power } \\
\text { Consumption }\end{array}$ & $\begin{array}{c}26 @ 75 \mathrm{~K} \\
1.0 @ 75 \mathrm{~K} \\
19.5 \mathrm{GHz} \\
2.0 \mathrm{GHz} \\
60 \mathrm{~m} \mathrm{~W} .\end{array}$ & $\begin{array}{c}30 @ 77 \mathrm{~K} \\
0.6 @ 77 \mathrm{~K} \\
19.5 \mathrm{GHz} \\
2.5 \mathrm{GHz} \\
>-30 \mathrm{dBm} \\
<60 \mathrm{~m} \mathrm{~W} .\end{array}$ \\
\hline Antenna & $\begin{array}{l}\text { Gain }(\mathrm{dB}) \\
\text { Sidelobes } \\
3 \text { dB beamwidth } \\
\text { Polarization } \\
\text { Feed loss }(\mathrm{dB})\end{array}$ & $\begin{array}{c}23 \\
-40 \mathrm{~dB} @ 30^{\circ} \\
15^{\circ} \\
\text { Circular } \\
<1.0\end{array}$ & $\begin{array}{c}26 \\
-40 \mathrm{~dB} @ 30^{\circ} \\
10^{\circ} \\
<0.5\end{array}$ \\
\hline Preselect & $\begin{array}{l}\text { Insertion loss } \\
3 \mathrm{~dB} \text { bandwidth } \\
\text { In-band ripple } \\
\text { Group delay }\end{array}$ & $\begin{array}{c}<0.5 \mathrm{~dB} \\
200 \mathrm{MHz} \\
<0.2 \mathrm{~dB}\end{array}$ & $\begin{array}{c}<0.3 \mathrm{~dB} \\
200 \mathrm{MHz} \\
5 \mathrm{n} \mathrm{sec}\end{array}$ \\
\hline Local OSC. & $\begin{array}{l}\text { Frequency }(\mathrm{GHz}) \\
\text { RF power }(\mathrm{dBm}) \\
\text { DC power }(\mathrm{mW}) \\
\text { Phase noise: } \\
@ 5 \mathrm{KHz} \text { offset } \\
@ 10 \mathrm{KHz} \text { offset } \\
@ 100 \mathrm{KHz} \text { offset } \\
@ 1 \mathrm{MHz} \text { offset }\end{array}$ & $\begin{array}{c}20.50<\mathrm{Fo}<20.90 \\
10.0 \\
90.0 \\
-57 \mathrm{dBC} / \mathrm{Hz} \\
-71 \mathrm{dBC} / \mathrm{Hz} \\
-97 \mathrm{dBC} / \mathrm{Hz} \\
-109 \mathrm{dBC} / \mathrm{Hz}\end{array}$ & $\begin{array}{l}-64 \\
-78 \\
-104 \\
-116\end{array}$ \\
\hline Mixer & $\begin{array}{l}\text { Noise figure }(\mathrm{dB}) \\
\text { Conversion loss } \\
1 \mathrm{~dB} \text { comp. point } \\
\text { Drive level } \\
\text { DC power }(\mathrm{mW})\end{array}$ & $\begin{array}{c}<6 @ 75 \mathrm{~K} \\
<6 @ 75 \mathrm{~K} \\
--10 \\
30\end{array}$ & $\begin{array}{c}<4 @ 75 \mathrm{~K} \\
<4 @ 75 \mathrm{~K} \\
>-20 \mathrm{dBm} \\
<30\end{array}$ \\
\hline Data rate & 1.024 Mbps BPSK & & \\
\hline
\end{tabular}


Table 2.-THERMAL BUDGET SHOWS COOLER CAN MEET MISSION REQUIREMENTS

\begin{tabular}{|c|c|c|c|}
\hline & Quantity & $\begin{array}{c}\text { Power/unit } \\
\mathrm{mW}\end{array}$ & Power mW \\
\hline Dc wires & 12 & 2 & 24 \\
\hline RF cables & 1 & 27 & 27 \\
\hline RF cables & 1 & 27 & 27 \\
\hline Support & & 50 & 50 \\
\hline MMIC & 1 & 60 & 60 \\
\hline Mixer & 1 & 30 & 30 \\
\hline Filter & 1 & 0 & 0 \\
\hline LO & 1 & 90 & 90 \\
\hline & & & 92 \\
\hline & & Total & 400 \\
\hline Available power & & & 400 \\
\hline
\end{tabular}

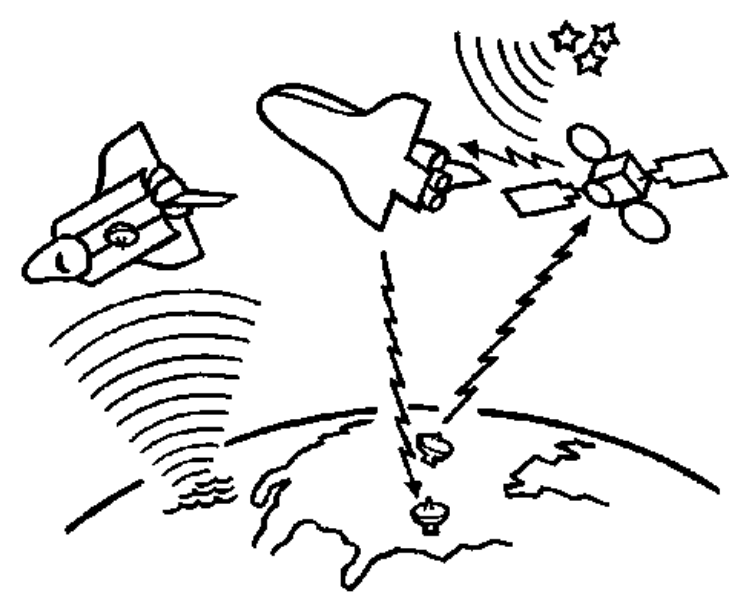

Figure 1.-LNR space experiment scenario showing essential orbiter attitudes, ACTS, and ground station at NASA Lewis and NASA Goddard. 


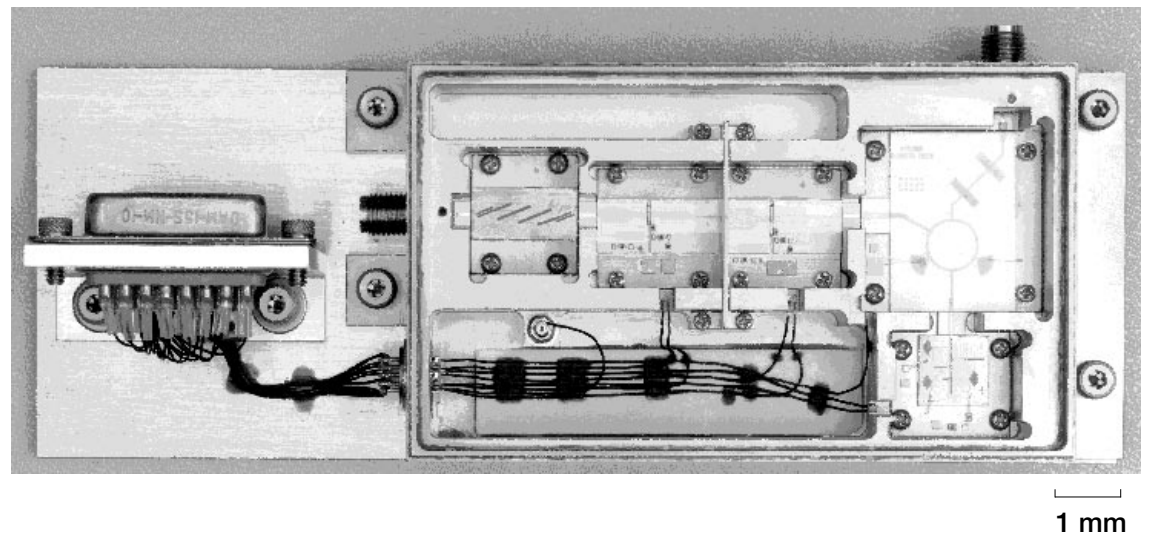

Figure 2.-X-band JPL/Lewis superconducting/semiconducting receiver delivered to NRL. The size is $5.5 \times 11.0 \times 1.6 \mathrm{~cm}^{3}$.

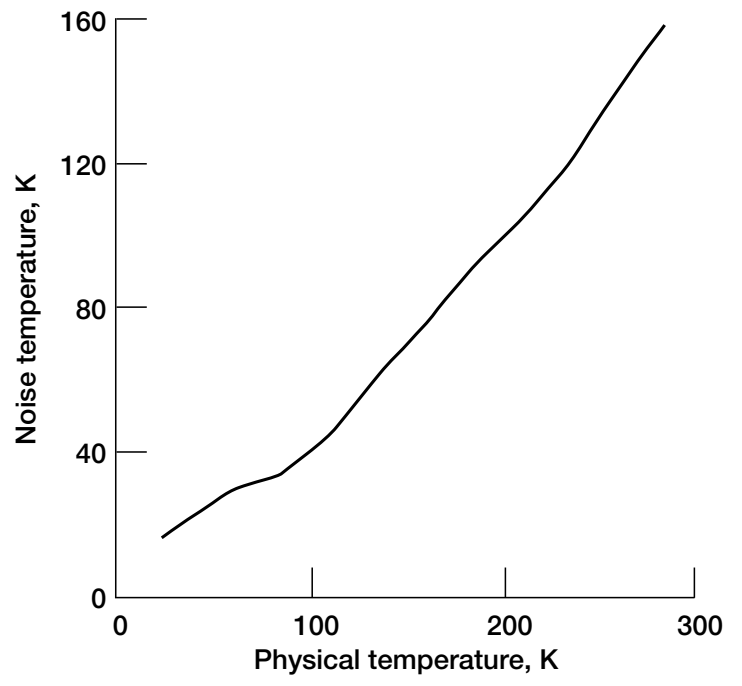

Figure 3.-20 GHz GaAs MMIC low noise amplifier noise temperature vs. temperature.

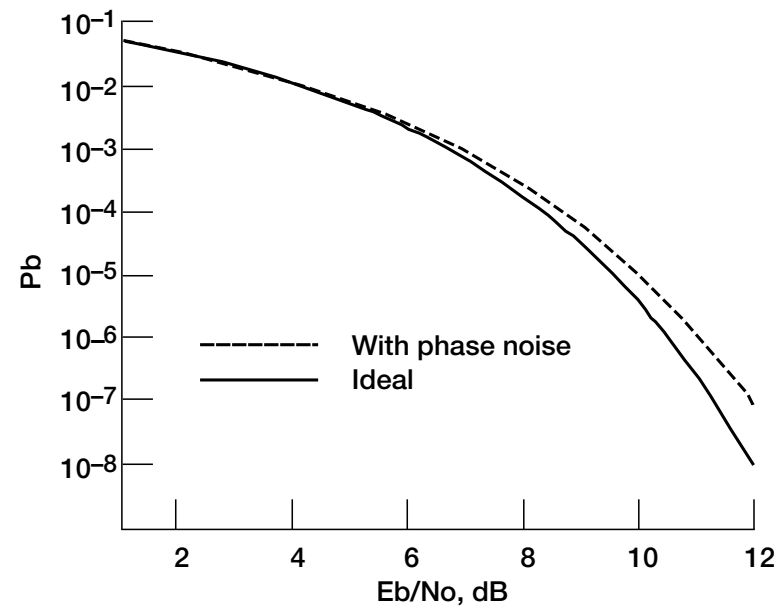

Figure 4.-Theoretical BER versus Eb/No for an ideal link and for a link corrupted by oscillator phase noise. Oscillator (loaded) Q is 250 . 


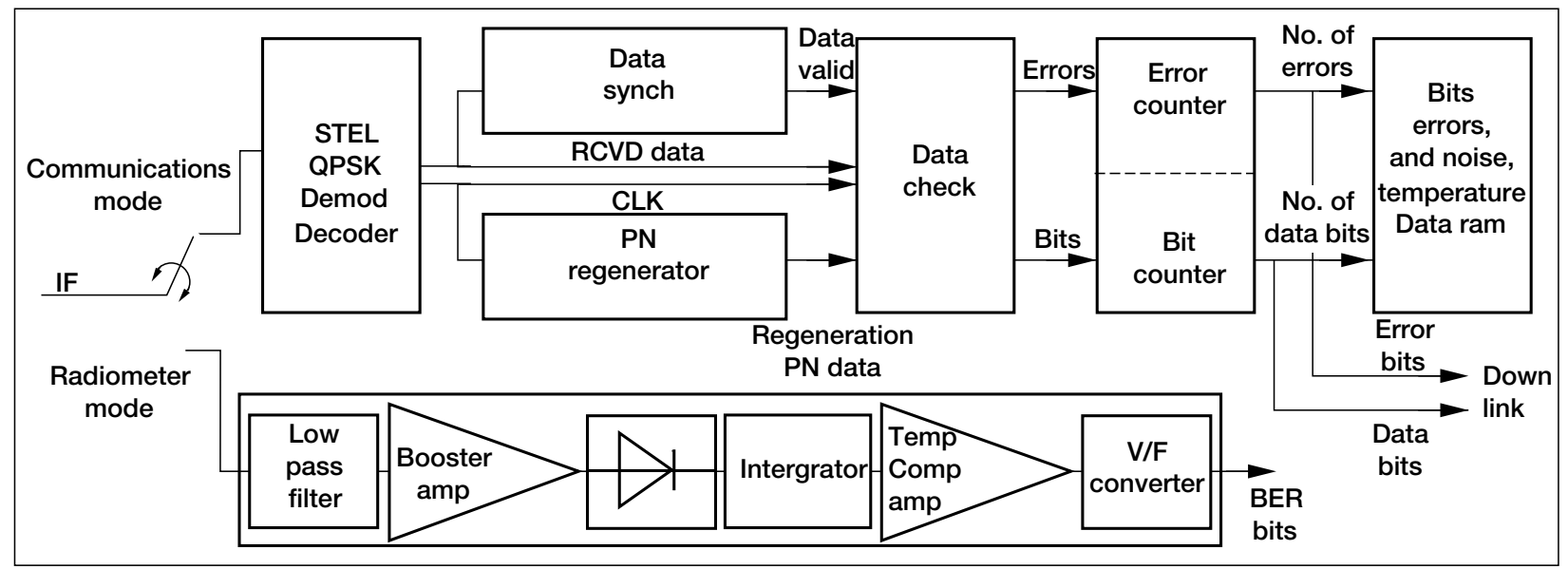

Figure 5.-Demodulator, BER counter, and radiometer block diagram. Additional filters and amplifiers preceed this section but are not shown. IF is $1.2 \mathrm{GHz}$.

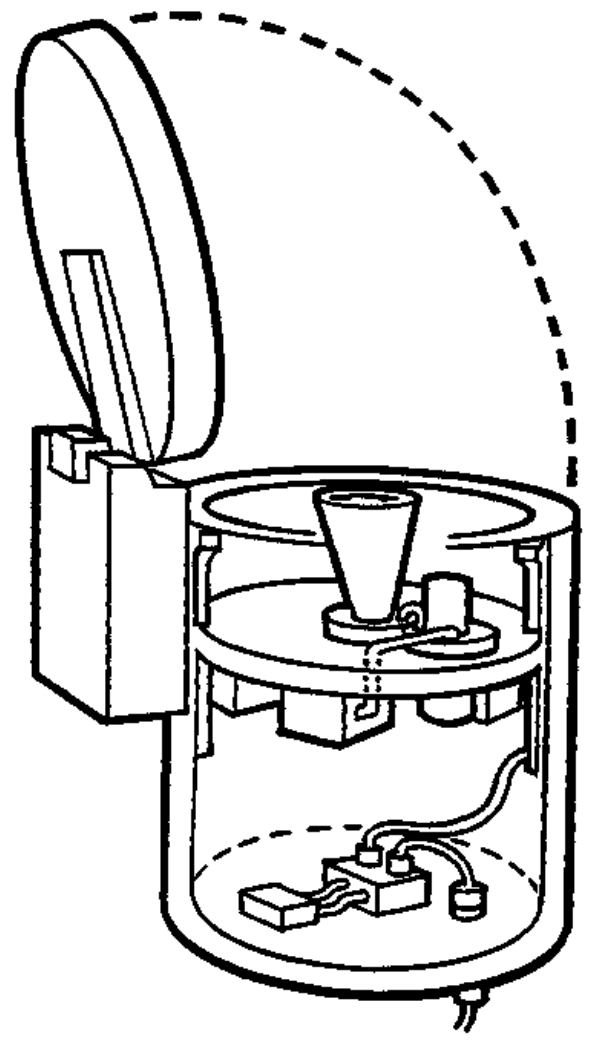

Figure 6.-Conceptual view of LNR and associated hardware in HitchHiker GAS can. The pulse tube cooler, horn antenna, downconverter, and signal processor are mounted to the top plate and shuttle interface equipment is at the bottom.

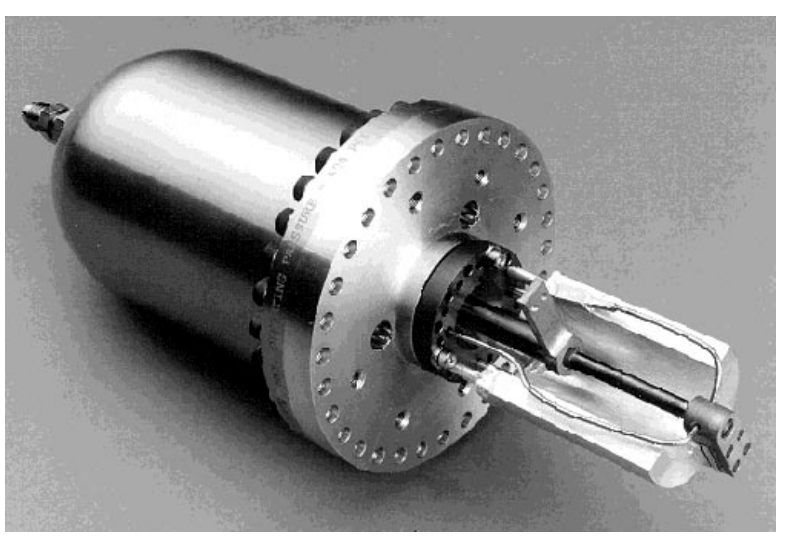

Figure 7.-The pulse tube cooler. The flange is about 4 inches in diameter and the cooler weighs about 4.7 pounds. 


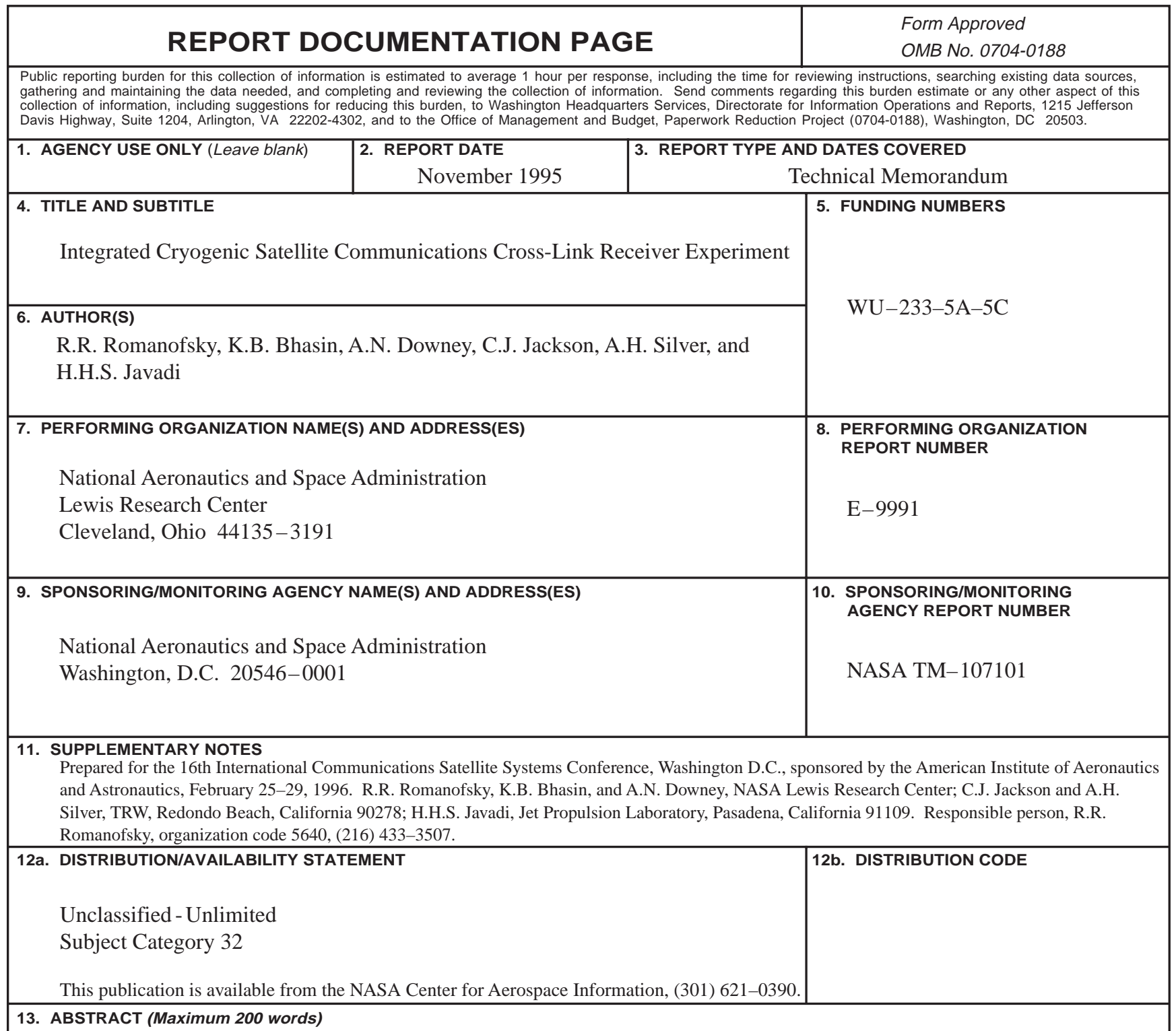

An experiment has been devised which will validate, in space, a miniature, high-performance receiver. The receiver blends three complementary technologies; high temperature superconductivity (HTS), pseudomorphic high electron mobility transistor (PHEMT) monolithic microwave integrated circuits (MMIC), and a miniature pulse tube cryogenic cooler. Specifically, an HTS band pass filter, InP MMIC low noise amplifier, HTS-sapphire resonator stabilized local oscillator (LO), and a miniature pulse tube cooler will be integrated into a complete $20 \mathrm{GHz}$ receiver downconverter. This cooled downconverter will be interfaced with customized signal processing electronics and integrated onto the space shuttle's "HitchHiker" carrier. A pseudorandom data sequence will be transmitted to the receiver, which is in low Earth orbit (LEO), via the Advanced Communication Technology Satellite (ACTS) on a $20 \mathrm{GHz}$ carrier. The modulation format is QPSK and the data rate is $2.048 \mathrm{Mbps}$. The bit error rate (BER) will be measured in situ. The receiver is also equipped with a radiometer mode so that experiment success is not totally contingent upon the BER measurement. In this mode, the receiver uses the Earth and deep space as a hot and cold calibration source, respectively. The experiment closely simulates an actual cross-link scenario. Since the receiver performance depends on channel conditions, its true characteristics would be masked in a terrestrial measurement by atmospheric absorption and background radiation. Furthermore, the receiver's performance depends on its physical temperature, which is a sensitive function of platform environment, thermal design, and cryocooler performance. This empirical data is important for building confidence in the technology.

14. SUBJECT TERMS

\begin{tabular}{|c|c|}
\hline & $\begin{array}{c}\text { 15. NUMBER OF PAGES } \\
13\end{array}$ \\
\hline & $\begin{array}{r}\text { 16. PRICE CODE } \\
\text { A03 }\end{array}$ \\
\hline $\begin{array}{l}\text { 19. SECURITY CLASSIFICATION } \\
\text { OF ABSTRACT } \\
\text { Unclassified }\end{array}$ & 20. LIMITATION OF ABSTRACT \\
\hline
\end{tabular}

Satellite; Communications; Cryogenic cooler; Receiver

\begin{tabular}{|c|c|}
\hline $\begin{array}{c}\text { 17. SECURITY CLASSIFICATION } \\
\text { OF REPORT }\end{array}$ & $\begin{array}{c}\text { 18. SECURITY CLASSIFICATION } \\
\text { OF THIS PAGE } \\
\text { Unclassified }\end{array}$ \\
Unclassified
\end{tabular}

NSN 7540-01-280-5500

Standard Form 298 (Rev. 2-89) Prescribed by ANSI Std. Z39-18 298-102 